\title{
The Importance of Next-generation Sequencing for Marine Larvae Research: Insight into Larvae Settlement and Anti-fouling
}

\author{
Kondethimmanahalli Chandramouli
}

Department of Diagnostic Medicine/Pathobiology, College of Veterinary Medicine, Kansas State University, Manhattan, KS 66506, USA

"Corresponding author: Kondethimmanahalli Chandramouli, Department of Diagnostic Medicine/Pathobiology, College of Veterinary Medicine, Kansas State University, Manhattan, KS 66506, USA, Tel: +785-473-3032; E-mail: khchandra@gmail.com

Received date: April 12, 2016; Accepted date: April 14, 2016; Published date: April 16, 2016

\section{Importance of Marine Invertebrates}

Larvae of marine invertebrates are important constituents of intertidal and sediment marine ecosystems around the world. They are ecologically and economically important species. For example, the barnacles are most dominant group of fouling organisms and are extensively used in larval settlement and anti-fouling research [1]. The bryozons and polychaetes are also cosmopolitan fouling organisms distributed in intertidal to shallow subtidal water $[2,3]$. These species form large colonies on ship hulls, piers and underwater structures causing a serious economic loss to shipping industries. It has been reported that biofouling of such species cost billions of dollars in shipping industry [4]. The academic and industrial marine research is mainly focused on biofouling species with the goal of understanding their larval settlement process and hoping for prevention of settlement and accumulation of such species on underwater structures. The life cycle of most marine invertebrates has two distinct stages: the pelagic larvae and the adults attached to marine substratum [5]. Understanding the larval settlement and attachment processes is important for prevention of biofouling. Most of the anti-fouling studies target larvae settlement process. However, process of settlement at molecular level is largely unknown due to limited genome information available for these species. In recent years, rapid technological advances in next-generation sequencers (NGS) have opened up possibilities to sequence larval transcriptome of marine invertebrates. Since NGS rapidly generates huge amount of sequence data in a very cost-effective way, larval biologists are now starting to integrate such sequencing methods into their research methods.

\section{Choice of NGS Platforms}

Sequencing platforms generates humongous data at reasonably less cost with increasing read lengths. However, a challenge currently larvae researcher facing is how to efficiently analyse the sequencing data. Are these tools producing meaningful data that helps to address the fundamental questions in larvae settlement biology research? In the beginning, our team and other researchers used 454 pyrosequencing platforms for producing transcriptome. Because of the difficulty in characterizing reads and assembly, many researches now consider Illumina platform is the better option. Recently, we produced a high coverage of transcriptome dataset obtained from paired-end Illumina sequences from barnacle larvae [6]. Currently, the RNA-Seq data produced by the Illumina assembly offered the best reference maps with the highest number of reads and contigs. Assemblies obtained by Illumina produce longer contigs and more gene annotations thereby yielding high coverage of the transcriptome. Some studies utilize both 454 and Illumina to maximize the number of annotations and minimize the error rate by correcting the 454 library with Illumina data [7].

\section{Application of NGS for Larvae Settlement and Anti- fouling Research}

In recent years, transcriptomic studies aiming to define the role of genes or transcripts during settlement of marine larvae are rapidly increasing. For example, in barnacle Balanus amphitrite, 454 pyrosequencing generated $\sim 630,000$ reads and assembly of reads led to identification of 23,451 contigs in cyprids larvae [8]. This study identified several transcripts involved in larval settlement pathways. The availability of this transcriptome dataset in public domains prompted several researchers to carryout investigations on barnacle genome. Yan et al. [9] carried out in silico data analysis of barnacle larvae transcriptome dataset to identify transcripts of neuropeptides/ peptide hormones and investigated their potential role in barnacle larval settlement. Another study, by using 454 platforms, characterized lectin-like isotigs and their involvement in barnacle larvae settlement [10]. In this study, the authors generated EST libraries containing stage specific contigs for each developmental stage. Lin et al. [11] used Illumina sequencer to generate $\sim 77$ million reads which produced $\sim 100,000$ contigs after assembly and annotation from tissue of prosoma and the base of the barnacle Tetraclita japonica formosana. Here the authors identified barnacle cement proteins that are involved in adhesion or attachment process. Recently, Wang et al. [12] reported the transcriptome of barnacle cement gland tissue and showed that expression of genes of cement proteins appears to be remains constant throughout the molt cycle. The above referred two studies offer valuable insight into growth and adhesion process during larvae molting and settlement. More recently, we constructed a transcript dataset of $\sim 150$ million reads and $\sim 90000$ contigs in nauplius larvae of barnacle from Red Sea [6]. Our study identified several stress responsive and osmoregulative transcripts which play a key role in developing larval adaptation and survival strategies in extreme marine environments. In the first transcriptome study of bryozon Bugula neritina, $\sim 120,000$ high-quality reads were assembled to produce nearly 6400 contigs [13]. This study demonstrated that integrated "OMICS" as a promising approach for accelerating the genomics studies in non-model marine species. In the follow-up study, the authors used Illumina HiSeq2000 platform to identify various genes involved in development, immune response and neurogenesis during developmental stages in the larvae of $B$. neritina [14]. We conducted integrated study of RNA sequencing and proteome analysis in the larval stages of polychaete Pseudopolydora vexillosa. We identified several transcripts and molecular processes that play a crucial role during larval-adult transition.

\section{Challenges in Marine Larval Genomics Research}

The substantial amount of sequence data from larvae is presenting considerable challenges. Our own studies identified huge numbers of 
Citation: Chandramouli K (2016) The Importance of Next-generation Sequencing for Marine Larvae Research: Insight into Larvae Settlement and Anti-fouling. J Data Mining Genomics \& Proteomics 7: e123. doi:10.4172/2153-0602.1000e123

Page 2 of 2

transcripts that doesn't match to reference genome datasets. The function such putative or hypothetical transcripts are often unknown. Those could be novel genes or specifically expressed in particular species or developmental stage. Novel data analysis pipelines are required to deduce the function of such transcripts. In order to develop broad spectrum anti-fouling compounds there is a need for commonly expressed transcripts in many biofouling marine species which meant that obtaining more sequencing data for other species. It will be difficult to explore the common function of genes found in such organisms. Bioinformatics analysis is the major the limiting factor in larval genome research. The data mining, assembly, annotation and visualization of thousands of transcripts are new concepts in larval research. So larvae biologists should aware of such pipelines for efficient analysis of the raw data.

\section{Future Perspective}

Transcriptomic studies cataloged hundreds of transcripts and revealed their possible association with molecular pathways of larval attachment. Such transcripts or genes may serve as potential targets for anti-fouling compounds. However, larval settlement is a complex process and it is rather challenging to build direct connection between the gene targets and anti-fouling compounds. The target genes should be validated by RNAi or knockouts methods that call for more studies at functional level. Further, the transcriptome represents only a small portion of genome so RNA sequencing doesn't provide the complete picture of genes structure and function. The future investigations should focus on whole genome sequencing. Finally, adapting efficient data mining pipelines for transcriptome or genome datasets increase the chance of obtaining information on gene regulation, DNA and protein interactions and epigenetics. This will enable larvae biologists to look into such areas for better understanding of larval settlement.

\section{References}

1. Aguirre J, Martin J, Braga J, Betzler C, Berning B, et al. (2008) Packed concentrations of sessile barnacles (Cirripedia: Sessilia) from the Early Pliocene of SE Spain. Facies 54: 193-206.

2. Cohen, Andrew N (2011) The Exotics Guide: Non-native Marine Species of the North American Pacific Coast. Center for Research on Aquatic
Bioinvasions, Richmond, CA, and San Francisco Estuary Institute, Oakland, CA.

3. Bastida-Zavala JR, Ten Hove HA (2002) Revisions of Hydroides Gunnerus, 1768 (Polychaeta: Serpulidae) from the Western Atlantic region. Beaufortia 52: 103-178.

4. Zapata M, Silva F, Luza, Y, Wilkens M, Riquelme C (2007) The inhibitory effect of biofilms produced by wild bacterial isolates to the larval settlement of the fouling ascidia Ciona intestinalis and Pyura praeputialis. Electron J Biotechnol 10: 149-159.

5. Hadfield MG (2000) Why and how marine-invertebrate larvae metamorphose so fast. Semin Cell Dev Biol 11: 437-443.

6. Chandramouli KH, Al-Aqeel S, Ryu T, Zhang H, Seridi L, et al. (2015) Transcriptomic and proteomic shift reveals mechanisms of adaptation in larvae of Red Sea barnacle Balanus amphitrite. BMC Genomics 16: 1063.

7. Ekblom R, Galindo J (2011) Applications of next generation sequencing in molecular ecology of non-model organisms. Heredity (Edinb) 107: 1-15.

8. Chen ZF, Chen ZF, Matsumura K, Wang H, Arellano SM, et al. (2011) Toward an understanding of the molecular mechanisms of barnacle larval settlement: a comparative transcriptomic approach. PLoS One 6: e22913.

9. Yan XC, Chen ZF, Sun J, Matsumura K, Wu RS, et al. (2012) Transcriptomic analysis of neuropeptides and peptide hormones in the barnacle Balanus amphitrite: evidence of roles in larval settlement. PLoS One 7: e46513.

10. De Gregoris TB, Rupp O, Klages S, Knaust F, Bekel T, et al. (2011) Deep sequencing of naupliar-, cyprid- and adult-specific normalised Expressed Sequence Tag (EST) libraries of the acorn barnacle Balanus amphitrite. Biofouling 27: 367-374.

11. Lin HC, Wong YH, Tsang LM, Chu KH, Qian PY, et al. (2014) First study on gene expression of cement proteins and potential adhesion-related genes of a membranous-based barnacle as revealed from Next-Generation Sequencing technology. Biofouling 30: 169-181.

12. Wang Z, Leary DH, Liu J, Settlage RE, Fears KP, et al. (2015) Moltdependent transcriptomic analysis of cement proteins in the barnacle Amphibalanus amphitrite. BMC Genomics 16: 859.

13. Wang H, Zhang H, Wong YH, Voolstra C, Ravasi T, et al. (2010) Rapid transcriptome and proteome profiling of a non-model marine invertebrate, Bugula neritina. Proteomics 10: 2972-2981.

14. Wong YH, Ryu T, Seridi L, Ghosheh Y, Bougouffa S, et al. (2014) Transcriptome analysis elucidates key developmental components of bryozoan lophophore development. Sci Rep 4: 6534. 